\title{
MATHEMATICAL QUESTIONS IN SEISMOLOGY
}

\author{
C. F. RICHTER
}

Seismologists, like mathematicians, are accustomed to dividing their subject into a pure and an applied branch. The desirability of such division is underlined by the manner in which non-seismologists frequently approach the subject. It is too often taken for granted that, because an earthquake is a motion of the ground, seismology must be chiefly concerned with the detailed analysis of that motion for its own sake. Such analysis is important in the engineering section of applied seismology; but it is unrepresentative of seismology as a whole, which deals largely with more remote problems: the causative mechanism of earthquakes, the distribution of their origins geographically and in depth, or the structure and physical condition of the interior of the earth.

Engineering applications are out of bounds for the present speaker. Briefly, they refer to the design of earthquake-resistant (not "earthquake-proof") structures. The problems differ from those of most structural engineering in being dynamical, not statical; buildings, bridges, and other structures must be designed with reference to their behavior when in violent vibratory motion. Further, horizontal forces must be considered more explicitly than is done in ordinary design, where the chief load forces, due to gravity, are vertical. Problems of principle are few; but the mechanical characteristics of large structures are only less complex than the motion of the ground in an earthquake. To arrive at solvable propositions it is usually necessary to idealize both sets of data to a degree which seriously limits the practical applicability. Consequently, progress has depended heavily on empirical investigation of the behavior of models on a shaking-table.

A very vocal offshoot of seismology is its application to geophysical prospecting - the investigation of subsurface structures by recording, with specially designed seismometers, the artificial earthquakes produced when explosives are detonated. Numerous books and periodicals deal with the problems of "geophysics"-which, in the vocabulary of the profession, means geophysical prospecting; a rather unwarranted restriction of the meaning of a necessary term, long in use with its proper significance as the physics of the earth. These problems present little of individual interest to the mathematician,

An address delivered before the meeting of the Society in Berkeley, Calif., on April 11, 1942 by invitation of the Program Committee; received by the editors June 29, 1942. 
since nearly all are specializations of problems and methods long familiar in connection with earthquakes. As an example, consider an idealization of "the reflection method." An explosion is set off at or near the surface of the earth, at a carefully recorded time. At unknown depth is a reflecting layer, of irregular form, which may be discontinuous in the mathematical sense. The arrival of elastic waves from the explosion, reflected at the named layer, is timed accurately at a number of points on the surface of the earth. Supposing the velocity known and constant, is the determination of arrival times at all points of a limited area (taken as plane) sufficient to determine the depth and form of the reflecting layer? More practically, what is the maximum information derivable from a limited number of observations? In practice, the explosions may be repeated at several different points.

An important division of seismology which as yet offers little hold to the mathematician is that dealing with the fundamental mechanisms of earthquakes - the possible physical origin of stresses giving rise to earthquakes, the mechanism of the actual process of fracturing. These problems belong to the field which has lately taken the name of "tectonophysics"; a section of the American Geophysical Union with that title was organized in 1939. This marks a movement to give greater precision, from the point of view of mathematical physics, to many of the concepts used in geology to describe the behavior of great masses of matter. The subject is being furthered by increasing experimental data on the physical properties of rocks and other material under great pressure. A detailed discussion would involve almost the whole of geophysics, of which seismology is only a small part. Reference may be made to the general manuals in the bibliography, and to the Transactions of the American Geophysical Union.

Theoretical seismology, like other branches of physics, is readily characterized mathematically by the occurrence of certain fundamental differential equations, the theory of which is involved in nearly every problem. These occur in three principal sets:

I. equations of motion of the recording instruments,

II. equations of motion of elastic solid bodies,

III. equations of propagation of elastic waves in the interior of the earth (usually assumed as spherical).

The first are the simplest. The motion of a seismometer is a particular case of the forced oscillations of vibrating systems, the theory of which is classical and well worked out. In its simplest form, the seismometer equation is a linear ordinary differential equation of the second order, with constant coefficients. The nonhomogeneous part 
represents the disturbance due to an earthquake. The homogeneous term of zero order determines the free period of oscillation of the seismometer pendulum, and that of first order then gives the damping, or rate of decrease of the free oscillation. Further complication occurs when the seismometer displacement, instead of being recorded directly, operates an electromagnetic system which actuates a recording galvanometer. The free period and damping of the galvanometer then enter in to the equations of motion of the coupled system, which in complete form are of fourth order. Since the coefficients are still constant, the problem is theoretically solved; but the practical application may become difficult and laborious if the damping coefficients are large enough to approach their critical values. Devices for clarifying the problem or shortening the computations are much to be desired.

Seismic waves are propagated through the whole earth; systematic investigation employs the data of many hundreds of earthquakes, as recorded at seismological stations in all parts of the formerly civilized world. The observations are affected by all ordinary causes of error, so that statistical treatment is necessary to ensure valid conclusions. Harold Jeffreys, at Cambridge, has devoted much of his distinguished ability for many years to the mathematical problems of geophysics and seismology; the professional mathematician will find no better introduction to these problems than his volume The earth [2]. Jeffreys' interest in the theory and technique of statistics as such has also appeared in many publications; in a recent volume [11] he has presented a detailed account of his somewhat unorthodox views. Seismologists have observed with much interest the application to their specialty of these highly refined techniques, which are for the most part beyond the range of their critical judgment. Jeffreys' specific results are mostly in excellent agreement with those of others who have not used his methods. He has sometimes claimed a surprisingly high degree of precision; but any difference of opinion on such points refers, not to the method of handling the data, but to the reliability of the observations as such. Others have attempted to reach the same objective by working so far as possible with original seismograms in preference to readings taken from them, and by selecting the data used for further conclusions through detailed consideration of each item, applying individual criteria not easily derived from a purely statistical treatment. Questions arise belonging not merely to statistics, but to the whole theory of scientific method. Reference may be made to Jeffreys' smaller volume [12], and to the excellent summary in Blake's paper [7]. 
Seismological data have at least one statistical idiosyncrasy; the errors are not symmetrical. The fundamental datum is the time of arrival of the first of a long succession of elastic waves at the recording station. If this first motion is small it may be missed, and a later time used instead. Early times, on the other hand, can arise only from the normally fluctuating causes of error, except occasionally when a seismologist, anxious not to overlook the true beginning, has measured a small extraneous disturbance preceding it.

The equations of set II are:

$$
\rho \frac{\partial^{2}(u, v, w)}{\partial t^{2}}=(\lambda+\mu) \operatorname{grad} \Theta+\mu \nabla^{2}(u, v, w),
$$

(2) $X_{x}=\lambda \Theta+2 \mu \frac{\partial u}{\partial x}, \quad Y_{y}=\lambda \Theta+2 \mu \frac{\partial v}{\partial y}, \quad Z_{z}=\lambda \Theta+2 \mu \frac{\partial w}{\partial z}$,

(3) $\quad X_{v}=\mu\left(\frac{\partial u}{\partial y}+\frac{\partial v}{\partial x}\right), \quad Y_{z}=\mu\left(\frac{\partial v}{\partial z}+\frac{\partial w}{\partial y}\right), Z_{x}=\mu\left(\frac{\partial w}{\partial x}+\frac{\partial \mu}{\partial z}\right)$.

$u, v, w$ are the cartesian components of displacement of any elementary particle from its equilibrium position. $\rho$ is the density; $\lambda$ and $\mu$ are the Lamé constants of elasticity. $\Theta$, the dilatation, is an abbreviation for the divergence of the vector $(u, v, w)$. Equation (1), which stands for three, is thus a vector equation; it suffices for the theory of motion of a homogeneous isotropic elastic solid of infinite extent, without boundaries or discontinuities. The remaining equations give the components of the stress tensor in terms of the derivatives of the displacements. These are called for whenever it is necessary to apply boundary conditions.

Consider first the consequences of these classical equations. Generalizations and modifications will be cited later. The usual process of separating the displacement vector into two parts, one of which is irrotational, involves taking the divergence and the curl of equation (1); this yields the two wave equations:

$$
\begin{gathered}
\frac{\partial^{2} \Theta}{\partial t^{2}}=\frac{\lambda+2 \mu}{\rho} \nabla^{2} \Theta, \\
\frac{\partial^{2}(\xi, \eta, \zeta)}{\partial t^{2}}=\frac{\mu}{\rho} \nabla^{2}(\xi, \eta, \zeta) .
\end{gathered}
$$

Here $2(\xi, \eta, \zeta)=\operatorname{curl}(u, v, w)$.

This process proves the possible existence of two types of elastic waves. In the first, displacements are purely compressional or dilata- 
tional, the displacement vector being in the direction of propagation of the wave. These are physically identical with sound waves. In the second type, displacement may be described as purely shearing, irrotational, or equivoluminal, being transverse to the direction of propagation and consequently exhibiting the phenomena of polarization as in optics. Whether these transverse waves should be called sound waves is a matter of terminology. Both longitudinal and transverse waves are observed in the earth. These are the only types of waves which can exist in an unlimited homogeneous isotropic elastic medium subject to equation (1). As soon as boundaries exist, however, other waves occur.

The simplest waves due to a boundary are the surface waves found by Lord Rayleigh [13]. Let the medium be semi-infinite with a free bounding surface at the plane $z=0$. Then a solution of (1) exists which contains a factor $\exp (a z+i b x-i c t)$ where $a, b, c$ are real. This represents a wave propagated in the direction of $x$ with velocity $c / b$; its amplitude decreases exponentially with increasing $z$ if $a$ is negative, so that on the positive side of the boundary the disturbance is practically confined to the vicinity of the free surface. The velocity $c / b$ is determined by requiring the stress components (2) and (3) to vanish at the boundary $z=0$. This leads to a cubic equation in $c^{2} / t^{2}$. This equation is obviously characteristically connected with the partial differential equation (1); an exact analysis of this type of relationship might be of much interest.

Since the cubic equation is reached by squaring, some of its roots may be extraneous to the problem. One of the roots is always appropriate, and leads to the solution given by Rayleigh. The case when the other two roots are equal falls well within the admissible numerical range of the physical constants, so that with slightly different possible values these roots may be either both real or both complex. When both are complex, they represent a valid solution of the given equations and boundary conditions only if the amplitude becomes infinite for infinite $z$; this is not of physical interest. When both roots are real, $a$ must be taken as imaginary; the solution is periodic and does not vanish at infinity. The velocities come out greater than those of the normal longitudinal and transverse waves represented by (4) and (5). This at first appears physically improbable. However, it readily appears that these solutions represent trains of plane longitudinal and transverse waves of the normal type and with the normal velocities, approaching the surface at specified angles; the high apparent velocity in the $x$ direction is due to these angles of emergence. The result then appears trivial; it seems that the search for solutions satisfying 
given conditions has led incidentally to a special case of another known solution. However, there is still a possible question: Does this mean physically that a disturbance below the plane free surface will actually produce, not merely the Rayleigh surface wave and the ordinary body waves with spherical wave front, but also these particular body waves with a different orientation? The same result was obtained by Somigliana [14] less directly, and has been applied by Caloi $[15,16]$ to explain certain observations of anomalously high velocities. To the present speaker it appears more probable that these observations have another explanation, and that the solutions in question correspond to no new physical phenomenon. The same case arises in the work of Jeans [26] to be cited presently.

The Rayleigh wave is only the simplest type of wave dependent on a discontinuity. Most seismograms show large waves of the type whose theory is due to A. E. H. Love [17]. These exist primarily in the space between two discontinuities; in the most important case the upper of these is the surface of the earth, while the lower is the base of the continental rocks. Similar waves may exist without a discontinuity, if the physical constants change rapidly with depth $[18,19]$. This shows that a complete theory, now lacking, of the propagation of elastic waves in a nonhomogeneous but isotropic medium, would include several types which do not occur when the medium is homogeneous and unbounded.

More general boundary conditions have been applied. Stoneley and others [20-25] have worked on the theory of waves associated with an internal interface, and on the effect of multiple layering. Jeans [26] has considered the general problem of free oscillations of a spherically symmetrical earth. Surface waves of Rayleigh and Love types, as well as others, appear as particular solutions; the general solution is not worked through. Lamb, Sezawa, Nakano and others [27-41] have developed the theory of generation of bodily and surface waves from an initial disturbance of given form; this bears directly on the problem of earthquake causation.

Earthquake surface waves show considerable dispersion. The simple Rayleigh theory gives no dispersion, the velocity being fixed; however, the velocity depends on the frequency for Love waves $[17,18]$ and for Rayleigh waves on the surface of a heterogeneous medium [42-49]. The observed dispersion differs regionally, and is evidence for regional differences in crustal structure [4, chap. XII].

A quite different problem of much importance in the interpretation of seismograms is the following: Plane waves, either longitudinal or transverse, are incident at a given angle on the plane boundary be- 
tween two media which differ in elastic constants and density. There are four derived plane waves; reflected longitudinal and transverse waves in the first medium, refracted longitudinal and transverse waves in the second medium. What is the ratio of the amplitude of each to that of the incident wave?

This problem was solved by Knott [50], and, a little more directly, by Zoeppritz [51]. The solution takes the form of four linear homogeneous algebraic equations in the amplitudes of the five waves, there being two cases according as the incident wave is longitudinal or transverse. Unfortunately, the coefficients are complicated, containing physical constants and trigonometric functions of the angles. Computation is laborious, and has been carried out only for a limited range of assumptions which approximate the actual conditions met with in seismology [52-54]. Our information is incomplete as to maxima and minima of the various amplitudes as functions of the physical parameters, and even as to the occurrence of zeros and singularities. It would appear that some of these questions might be dealt with directly from the equations of motion and the boundary conditions in differential form; but this has not been done.

On seismograms of earthquakes at distances between 100 and 1000 kilometers, the direct longitudinal wave, $\bar{P}$, is preceded by a longitudinal wave $P_{n}$, refracted through the material beneath the continental layers in which most earthquakes originate. At that depth it travels so fast that it arrives ahead of the direct wave. Analogous waves refracted horizontally through subsurface layers are observed in geophysical prospecting. Simplified forms of the theory usually indicate that such a horizontally refracted wave should exist as a type of surface wave in the lower medium, but that it should carry only a small part of the incident energy. While the direct wave is in fact larger, the observed refracted wave is usually not so small as the simple theory suggests. More precise discussion, such as that of Jeffreys $[55,56 ; 57$, pp. 219-220] and Muskat [58] still does not satisfactorily represent the observations. Alternative explanations have been looked for. Recently O. von Schmidt $[59,60]$ has succeeded in producing essentially the same phenomenon in the laboratory. He emphasizes the analogy with the "head wave" in ballistics. A theory has been given by Joos and Teltow [61], but the subject is still incompletely investigated.

In practice, the equations of our set III are identical with the fundamental equations of geometrical optics. The dimensions of the earth and its larger structures are so vast compared with the wave lengths of bodily seismic waves (mostly of the order of ten kilometers) that 
the observations can be discussed without much attention to phenomena of interference or diffraction. The chief difference from geometrical optics lies in the data, the more significant of which are determinations of time, whereas intensities and frequencies, though observed and used, are of only supplementary importance.

The customary general statement is Fermat's "principle of least time"

$$
\delta \int d s / v=0
$$

where the end points of the line integral are fixed during the variation. $d s$ denotes the element of path, and $v$ the velocity of propagation given as a function of position. The name is slightly misleading. Thus, when a shock originates within the earth and sends waves to a distant surface point, there are two principal paths of singly reflected waves satisfying the variation principle. Of these the more obvious and familiar, reflected nearly midway, corresponds to a maximum of the integral. The other, representing a minimum time, is reflected comparatively near the source; it is observed chiefly in earthquakes originating at great depth. Jeffreys $[73$, p. 560] has pointed out that in general seismic waves corresponding to maximum times are less sharp and well defined on the seismograms than those with minimum times.

Attention is called to the differential equation associated with the variation principle, and named by Bruns the equation of the "eikonal." For a spherically symmetrical earth it takes the form

$$
(\partial t / \partial r)^{2}+\left(1 / r^{2}\right)(\partial t / \partial \theta)^{2}=1 / v^{2}
$$

where $r, \theta, \phi$ are polar coordinates with origin at the center and polar axis through the source of the earthquake. Since the velocity $v$ is independent of $\phi$, that coordinate drops out. $t$ is the time of propagation, not along a fixed ray, but always along that ray which forms the path of least time from the source to the point $(r, \theta, \phi)$.

This general equation can be made the basis for a complete discussion of the geometrical optics of seismology. More customarily, the wanted information is derived from a special consequence, namely Snell's law of refraction in its polar form, which states that along a given ray

$$
(r \sin i) / v=\text { const. }=r_{s} / v_{s} .
$$

Here $i$ is the angle between the radius vector and the tangent to the ray. Except at the boundary of the core of the earth, velocity in- 
creases with depth (since the elasticity increases more rapidly than the density, which alone would give the opposite effect). Seismic rays are convex toward the center of the earth, and each ray has a minimum radius vector $r_{s}$ corresponding to $i=90^{\circ}$; the velocity at this point on the ray is $v_{s}$.

The initial problem is to determine the velocity distribution within the earth. The observed times at seismological stations at varying angular distances from the source give $t$ as a function of $\theta$ for $r=R$, the radius of the earth. The assumption of spherical symmetry is justified by the satisfactory agreement with all observations of tables giving $t$ as a function of $\theta$ alone. The determination of $v(r)$ from $t(\theta)$ involves a Fredholm integral equation, of the type first solved by Abel [62, 63]. The method developed by Wiechert [64] and Herglotz [65], improved by Bateman [66], leads to integrals which are readily evaluated graphically or with a computing machine. The essential equations are:

$$
\begin{aligned}
\cosh q(x, \theta) & =t^{\prime}(x) / t^{\prime}(\theta), \\
\log \frac{R}{r_{s}} & =\frac{1}{\pi} \int_{0}^{\theta} q(x, \theta) d x, \\
r_{s} & =v_{s} t^{\prime}(\theta) .
\end{aligned}
$$

From the given $t(\theta)$ one proceeds to $t^{\prime}(\theta)$ and thence to $v_{s}$ as a function of $r_{s}$, identical with $v(r)$. General relations between the derivatives and singularities of $v(r)$ and $t(\theta)$ are of importance; few have been worked out. ${ }^{1}$ A peculiar singularity is involved, which occurs in all similar problems. To state it simply, consider a source of disturbance at $x=0, y=0, z=-h$, the velocity $v$ being a function of $z$ only. Let the arrival time on the $x$-axis be given as $t(x)$. Then $t^{\prime}(x)=0$ for $x=0$, so long as $h$ is different from zero; but if $h=0$ then $t^{\prime}(x)=1 / v$ for $x=0$. Again, if $h$ is not zero, $t^{\prime \prime}(x)=0$ for $x=0$; but it is not easily seen what the general behavior of the second derivative will be if $h=0$. This apparently artificial problem took on a physical significance in the following way $[9$, IV; 67,68$]$ : There is a well observed type of seismic wave which originates as a transverse wave, continues along a short path in the presumably liquid core of the earth as a longitudinal wave, and then emerges from the core as a transverse wave, in which form it reaches the recording stations. Shortening dis-

${ }_{1}^{1}$ The speaker gave the result by Gutenberg and Richter $[9$, II $]$ for $d v_{s} / d r_{s}$ in terms of $t^{\prime}$ and $q$. Subsequent discussion has made possible an adequate discussion of the higher derivatives, to be published in the near future. Correction to $[9, I I$, p. 304]: $d v_{s} / d r_{s}=0$ for $I=\pi$, not for $I$ infinite. 
tance between earthquake source and observing station shortens the path in the core, until it finally vanishes and the wave in question ceases to exist. Let $t(\theta)$ now represent the times of arrival at the earth's surface; what should be the behavior of $t^{\prime \prime}(\theta)$ at the minimum distance? The question bears on the expected amplitude of the given wave at this minimum distance. Considering the path in the core leads to the same singularity as in the simple plane case just described. Dix [69] has shown that the second derivative should vanish except for certain highly singular velocity distributions.

Some large scale diffraction phenomena occur. The core of the earth, which has a relatively sharp boundary at a depth of 2900 kilometers, does not transmit transverse waves; and at the boundary the velocity of longitudinal waves drops from about $13 \mathrm{~km} / \mathrm{sec}$ outside to about $8 \mathrm{~km} / \mathrm{sec}$ inside; this is the only noteworthy exception to the rule of increasing velocity with depth. There results a shadow zone for both types of waves, within which disturbances diffracted around the core are found on the seismograms of sensitive instruments recording great earthquakes. Further, there is an angle of minimum deviation for the principal longitudinal waves refracted through the core; this angle determines the outer limit of the shadow zone, which accordingly exhibits the phenomenon known in optics as a caustic, with a focussing effect producing large amplitudes in a small range of $\theta$. Waves apparently continuous with the others are observed irregularly within the outer limit. For years these were attributed to diffraction associated with the caustic-more precisely, with its continuation as a caustic surface within the earth; but no exact theory was attempted until quite recently [70]. Meanwhile, it has appeared that the observations are better explained by the existence of an "inner" core, the boundary of which need not be very sharp [71-73].

Many generalizations have been aimed at extending the applicability of the theory. Thus, we have handled "optical" seismology assuming a spherical earth. The effects of ellipticity are definitely observable; indeed, it has been suggested [74, p. 231] that seismological data would suffice for a rough determination of the oblateness of the terrestrial spheroid. A good beginning has been made in these problems [75-79], which fall into three evident stages: a figure-of-the-earth problem to indicate the probable variation in ellipticity with depth for the homogeneous shells constituting the earth; a solution for the propagation of elastic waves through such a body; and a detailed examination of this solution for new phenomena of reflection and refraction not possible with spherical symmetry. 
Love [17] and later writers have investigated the effect of gravity on the propagation of seismic waves; this proves to be entirely negligible.

A difficult series of papers by Uller [80] offers a generalization of the wave concept as such, with seismological applications. Further critical discussion is needed. Summaries have been given by Macelwane [3, pp. 110-114) and Gutenberg [1, vol. 4, pp. 136-146].

Some extension of theory is required to account for the frequent observation that the prevailing periods of all types of seismic waves increase with distance. This cannot wholly be due to the normal spreading of an impulsive disturbance with filtering out of the shorter periods. For surface waves, where very long paths are observable, often up to several complete circuits of the earth, such an explanation can be ruled out. Surface waves show dispersion, the theory showing that velocity should vary with period; but this cannot account for the increase with distance of the period of a clearly identifiable wave group. For body waves the corresponding evidence is less conclusive, but convincing to anyone familiar with the data. The required effect will be given by some type of viscosity, or internal friction. Sezawa [81-83] has set up a theory which partially covers the case; the problem has been treated by Jeffreys $[84,85 ; 2$, pp. 265-266], and by Gutenberg and Schlechtweg [86].

Confusion, especially when there is insistence on carrying on the discussion in apparently non-mathematical terms, arises from the use of such terms as "viscosity" and "strength" in different senses. Definition of viscosity for solids is difficult, depending on the form of the hypothetically modified equations of elasticity. The property most closely corresponding to the well known and well defined viscosity of fluids is commonly termed internal friction; the entirely different quantity which measures the slow yielding of solids under long-continued pressure is more usually termed viscosity. For discussion see Gutenberg [4, chap. XV].

The classical theory of elasticity requires the components of stress to be linear functions of those of strain. This first-order approximation is being extended by second-order theories. To this end Murnaghan's theory of finite strain [87] may be applied, assuming "constants" to be modified in a medium under strain, the case of most importance being that of hydrostatic pressure. On this basis Birch [89] has found close agreement between observed and calculated seismic wave velocities in the earth, and has shown [88] that initial hydrostatic pressure does not affect the laws of elastic wave propagation. Biot [90] has developed a general second-order theory, and has recently [91] ap- 
plied it to the general problem of propagation of elastic waves when there is initial stress; hydrostatic pressure produces no effect, but other types of initial stress may lead, among other effects, to a coupling between longitudinal and transverse waves. The non-effectiveness of hydrostatic pressure restricts the applicability of the old results of Rudzki [92], who assumed the earth to be aeolotropic, with different wave velocities horizontally and vertically. He arrived at the same coupling of longitudinal and transverse waves; but his theory gave no ready means of estimating the magnitude of the supposed seismological effect.

Mathematical seismology is much in need of synthesis. Techniques to solve particular problems have been imported from other applications or from pure mathematics, without much examination of their relation to each other and to the general fields in which they originate. Hence the implications of such special results as the cubic equation characterizing the Rayleigh wave, or the Abelian integral equation in the velocity problem, are often imperfectly understood by those who are constantly working with them. Not rarely this leads to difficulties when the problems are to be generalized. Much spade work remains in exploration of the various subjects suggested by the natural articulation of the data; but the ultimate outcome should be a comprehensive treatment akin to the great treatises on optics and acoustics. To repeat-the contents of those treatises have only a limited application in seismology, not merely because of the complication in combining the mathematical characteristics of optics and acoustics, but largely because the principal observed data are not frequencies and intensities, but actual times of propagation of seismic waves.

It would be ungracious to close without reference to the interesting address by Professor Cairns [6], who has since been a welcome and stimulating visitor at the Pasadena laboratory.

In preparing for publication, much use has been made of the valuable summary by Blake [7]; his discussion and references cover a number of points not included here. The paper by Byerly [8] contains further supplementary material.

The following bibliography is selected for general usefulness or for mathematical interest. Many important papers, particularly those reporting observations, are omitted. Less than justice is done to the contributions by Jeffreys; more will be found in the Geophysical Supplement to the Monthly Notices of the Royal Astronomical Society. (Each volume of the Supplement covers a number of years; dates given are those of publication.) Further papers by Sezawa or by Sezawa and Kanai, as well as by other Japanese authors, will be 
found in the Bulletin of the Earthquake Research Institute of Tokyo Imperial University. Japanese papers often are of mathematical interest only, by reason either of initial assumptions too remote from physical fact, or of failure to carry the calculations far enough for comparison with observations. All those cited here are in English. Throughout the bibliography, the language of the title as given is that of the original paper.

\section{BIBLIOGRAPHY}

\section{A. Treatises AND General Papers}

1. B. Gutenberg, edited by, Handbuch der Geophysik, Berlin, Vieweg, 1929-1936. (Especially vol. IV, Erdbeben.)

2. H. Jeffreys, The earth, Cambridge, 2d edition, 1929.

3. J. B. Macelwane, edited by, Seismology (Bulletin of the National Research Council), Physics of the earth. VI, Washington, National Research Council, 1933. (Theoretical sections by Macelwane and Byerly.)

4. B. Gutenberg, edited by, Internal constitution of the earth (Bulletin of the National Research Council), Physics of the earth. VII, New York, 1939. (Seismological sections by Macelwane and Gutenberg.)

5. J. B. Macelwane and F. W. Sohon, Introduction to theoretical seismology. New York, Part I, Geodynamics (Macelwane), 1936; Part II, Seismometry (Sohon), 1932. (Mathematical processes given in full elementary detail.)

6. W. D. Cairns, Seismology from a mathematical view-point, Science vol. 89 (1939) pp. 113-118.

7. A. Blake, Mathematical problems in seismology, Transactions of the American Geophysical Union 1940 pp. 1094-1113.

8. P. Byerly, A seismologist's difficulties with some mathematical theory or the lack of $i t$, ibid. pp. 1113-1118.

9. B. Gutenberg and C. F. Richter, On seismic waves. I, II, III, IV, Gerlands Beiträge zur Geophysik vol. 43 (1935) pp. 56-133; vol. 45 (1935) pp. 280-360; vol. 47 (1936) pp. 73-131; vol. 54 (1939) pp. 94-136.

10. A. E. H. Love, $A$ treatise on the mathematical theory of elasticity, Cambridge, 4th edition, 1927. (The standard manual.)

\section{B. Special References}

11. H. Jeffreys, Theory of probability, Oxford, 1939.

12. - Scientific inference, Cambridge, 1937.

13. Lord Rayleigh (John W. Strutt), On waves propagated along the plane surface of an elastic solid, Proc. London Math. Soc. (1) vol. 17 (1885) pp. 4-11; Scientific papers, Cambridge, 1900, vol. 2, pp. 441-447.

14. C. Somigliana, Sulla propagazione delle onde sismiche, Rendiconti della Reale Accademia dei Lincei, Classe di scienzie fisiche, mathematiche e naturale (5) vol. 26 (1917) pp. 369-381, 472-480; vol. 27 (1918) pp. 13-20.

15. P. Caloi, Due novi tipi di onde sismiche alla luce di una teoria del Somigliana, ibid. (6) vol. 23 (1936) pp. 507-511.

16. - Nuova onda a lungo periodo oscillante nel piano principale; sue caratteristiche e confronto con l'onda $G$, Bollettino del Comitato per la Geodesia e la Geofisica del Consiglio Nazionale delle Richerche (2) vol. 6 (1936); reprint repaged 1-14. 
French translation in Publications du Bureau Central séismologique International (A) Travaux scientifiques vol. 15 (1937) pp. 93-106.

17. A. E. H. Love, Some problems of geodynamics, Cambridge, 1911; reprinted 1926.

18. E. Meissner, Elastische Oberflächenwellen mit Dispersion in einem inhomogenen Medium, Vierteljahresschrift der naturforschenden Gesellschaft in Zürich vol. 66 (1921) pp. 181-195.

19. —, Elastische Oberflächen-Querwellen, Verhandlungen der zweiten Kongress für technische Mechanik in $Z$ ürich 1926 (published at $Z$ ürich, 1927) pp. 3-11.

20. R. Stoneley, Elastic waves at the surface of separation of two solids, Proc. Roy. Soc. London Ser. A vol. 106 (1924) pp. 416-428.

21. - Love waves in a triple surface-layer, Monthly Notices of the Royal Astronomical Society (London), Geophysical Supplement vol. 4 (1937) pp. 43-50.

22. R. Stoneley and E. Tillotson, The effect of a double surface layer on Love waves, ibid. vol. 1 (1928) pp. 521-527.

23. K. Sezawa and G. Nishimura, Rayleigh-type waves propagated along an inner stratum of a body, Bulletin of the Earthquake Research Institute, Tokyo Imperial University vol. 5 (1928) pp. 85-91.

24. K. Sezawa and K. Kanai, The formation of boundary waves at the surface of a discontinuity within the earth's crust. I, II, ibid. vol. 16 (1938) pp. 504-526; vol. 17 (1939) pp. 539-546.

25. - The range of possible existence of Stoneley-waves, and some related problems, ibid. vol. 17 (1939) pp. 1-8.

26. J. H. Jeans, The propagation of earthquake waves, Proc. Roy. Soc. London Ser. A vol. 102 (1923) pp. 554-574.

27. H. Lamb, On the propagation of tremors over the surface of an elastic solid, Philos. Trans. Roy. Soc. London Ser. A vol. 203 (1904) pp. 1-42.

28. A. E. H. Love, The propagation of wave-motion in an isotropic elastic solid, Proc. London Math. Soc. (2) vol. 1 (1904) pp. 291-344.

29. H. Nakano, On Rayleigh wave, Japanese Journal of Astronomy and Geophysics vol. 2 (1925) pp. 233-326.

30. Win Inouye, Notes on the origin of earthquakes. I, II, III, IV, V, Bulletin of the Earthquake Research Institute, Tokyo Imperial University vol. 14 (1936) pp. 582597; vol. 15 (1937) pp. 90-100, 674-685, 686-696, with revisions on pp. 956-964; vol. 16 (1938) pp. 125-130.

31. G. Nishimura, On the elastic waves due to pressure variation on the inner surface of a spherical cavity in an elastic solid, ibid. vol. 15 (1937) pp. 614-635.

32. G. Nishimura and Takeo Takayama, Seismic waves due to tractions applied to the inner surface of a spherical cavity in an elastic earth, ibid. vol. 16 (1938) pp. 317-353.

33. K. Sezawa, Dilatational and distortional waves generated from a cylindrical or spherical origin, ibid. vol. 2 (1927) pp. 13-20.

34. - Periodic Rayleigh-waves caused by an arbitrary disturbance, ibid. vol. 7 (1929) pp. 193-206.

35. - Generation of Rayleigh-waves from an internal source of multiplet type, ibid. vol. 7 (1929) pp. 41-63.

36. - Love waves generated from a source of a certain depth, ibid. vol. 13 (1935) pp. 1-17.

37. K. Sezawa and K. Kanai, Amplitudes of $P$ and $S$ waves at different focal distances, ibid. vol. 10 (1932) pp. 299-308. 
38. - Polarization of elastic waves generated from a plane source, ibid. vol. 14 (1936) pp. 490-504.

39. - Transmission of arbitrary elastic waves from a spherical source, solved with operational calculus, ibid. vol. 19 (1941) pp. 151-160.

40. H. Kawasumi, Study on the propagation of seismic waves. I, II, ibid. vol. 11 (1933) pp. 403-452; vol. 12 (1934) pp. 660-703, with correction on pp. 854-856.

41. - An historical sketch of the development of knowledge concerning the initial motion of an earthquake, Publications du Bureau Central Séismologique International (A) Travaux scientifiques vol. 15 (1937) pp. 258-330. (Very complete, with extensive bibliography.)

42. K. Sezawa, Dispersion of elastic waves propagated on the surface of stratified bodies and on curved surfaces, Bulletin of the Earthquake Research Institute, Tokyo Imperial University vol. 3 (1927) pp. 1-18.

43. - - A kind of waves transmitted over a semi-infinite solid body of varying elasticity, ibid. vol. 9 (1931) pp. 310-315.

44. —_, Anomalous dispersion of elastic surface waves, ibid. vol. 16 (1938) pp. 225-232. Additional note, pp. 525-526.

45. K. Sezawa and K. Kanai, Anomalous dispersion of elastic surface waves, ibid. vol. 16 (1938) pp. 683-689.

46. - Discontinuity in the dispersion curves of Rayleigh waves, ibid. vol. 13 (1935) pp. 237-243.

47. T. Suzuki, Amplitude of Rayleigh waves on the surface of a stratified medium, ibid. vol. 11 (1933) pp. 187-194.

48. R. Stoneley, The transmission of Rayleigh waves in a heterogeneous medium, Monthly Notices of the Royal Astronomical Society (London), Geophysical Supplement vol. 3 (1934) pp. 222-232.

49. C. L. Pekeris, The propagation of Rayleigh waves in heterogeneous media, Physics vol. 6 (1935) pp. 133-138; an addition, p. 178.

50. C. G. Knott, Reflexion and refraction of elastic waves, with seismological applications, London, Edinburgh and Dublin Philosophical Magazine and Journal of Science (5) vol. 48 (1899) pp. 64-97.

51. K. Zoeppritz, Ueber Erdbebenwellen VIIB: Ueber Reflexion und Durchgang seismischer Wellen durch Unstetigkeitsflächen, Nachrichten von der königlichen Gesellschaft der Wissenschaften zu Göttingen, Mathematisch-physikalische Klasse, 1919, pp. 66-84.

52. $\mathrm{H}$ Blut, Ein Beitrag zur Theorie der Reflexion und Brechung elastischer Wellen an Unstetigkeitsflächen. I, II, Zeitschrift für Geophysik vol. 8 (1932) pp. 130-144, 305-322.

53. L. B. Slichter and G. V. Gabriel, Studies in reflected seismic waves. I, Gerlands Beiträge zur Geophysik vol. 38 (1933) pp. 228-238 (Summarizes earlier work.)

54. L. B. Slichter, Studies in reflected seismic waves. II, ibid. vol. 38 (1933) pp. 239256. Correction, vol. 43 (1935) p. 331.

55. H. Jeffreys, On compressional waves in two superposed layers, Proc. Cambridge Philos. Soc. vol. 23 (1926) pp. 472-481.

56. - The formation of Love waves (Querwellen) in a two-layer crust, Gerlands Beiträge zur Geophysik vol. 30 (1931) pp. 336-350.

57. — - A further study of near earthquakes, Monthly Notices of the Royal Astronomical Society (London), Geophysical Supplement vol. 4 (1937) pp. 196-225.

58. M. Muskat, Theory of refraction shooting, Physics vol. 4 (1933) pp. 14-28. 
59. O. von Schmidt, Ueber Kopfwellen in der Seismik, Zeitschrift für Geophysik vol. 15 (1939) pp. 141-148.

60. - Ueber Knallwellenausbreitung in Flilssigkeiten und festen Körpern, Zeitschrift für technische Physik vol. 19 (1938) pp. 554-561.

61. G. Joos and T. Teltow, Zur Deutung der Knallwellenausbrietung an der Trennschicht zweier Medien, Physikalische Zeitschrift vol. 40 (1939) pp. 289-293.

62. N. H. Abel, Solution de quelques problèmes a l'aide d'integrales définies, Oeuvres complètes de Niels Henrik Abel (Sylow and Lie, edited by), Christiania, 1881, vol. 1 pp. 11-27. From Norwegian original in Magazin for Naturvideskaberne vol. 1 (1823).

63. - A Aflösung einer mechanischen Aufgabe, J. f. reine u. angew. Math. vol. 1 (1826) pp. 153-157. French version in Oeuvres complètes, vol. 1 pp. 97-101.

64. E. Wiechert, Ueber Erdbebenwellen I: Theoretisches iber die Ausbreitung der Erdbebenwellen, Nachrichten von der königlichen Akademie der Wissenschaften zu Göttingen, Mathematisch-physikalische Klasse, 1907, pp. 415-529.

65. G. Herglotz, Ueber das Benndorfsche Problem der Fortpflanzungsgeschwindigkeit der Erdbebenstrahlen, Physikalische Zeitschrift vol. 8 (1907) pp 145-147.

66. $\mathrm{H}$. Bateman, The solution of the integral equation which connects the velocity of propagation of an earthquake wave in the interior of the earth with the times which the disturbance takes to travel to different stations on the earth's surface, London, Edinburgh and Dublin Philosophical Magazine and Journal of Science (6) vol. 19 (1910) pp. 576587. German translation in Physikalische Zeitschrift vol. 11 (1910) pp. 96-99.

67. B. Gutenberg, On focal points of $S K S$, Bulletin of the Seismological Society of America vol. 28 (1938) pp. 197-200.

68. I. Lehmann, On $S K S$, Geodaetisk Institut (Copenhagen) Meddelelse no. 15 (1940) pp. 1-14.

69. C. H. Dix, Derivatives of travel-time curves, Bulletin of the Seismological Society of America vol. 30 (1940) pp. 25-26.

70. Jean Coulomb, Diffraction d'un ebranlement au voisinage d'une caustique: application aux ondes $P^{\prime}$, ibid. vol. 30 (1940) pp. 27-34.

71. I. Lehmann, $P^{\prime}$, Publications du Bureau Central Stismologique International (A) Travaux scientifiques vol. 14 (1936) pp. 87-115.

72. B. Gutenberg and C. F. Richter, $P^{\prime}$ and the earth's core, N.onthly Notices of the Royal Astronomical Society (London), Geophysical Supplement vol. 4 (1938) pp. 363-372.

73. H. Jeffreys, The times of the core waves. I, II, ibid. vol. 4 (1939) pp. 548-561, $594-615$.

74. - Further corrections to the $P, S$, and SKS tables, ibid. vol. 4 (1937) pp. $225-250$.

75. - On the ellipticity correction in seismology, ibid. vol. 3 (1935) pp. 271274.

76. K. E. Bullen, The variation of density and the ellipticities of strata of equal density within the earth, ibid. vol. 3 (1936) pp. 395-401.

77. - The ellipticity correction to travel-times of $P$ and $S$ earthquake waves, ibid. vol. 4 (1937) pp. 143-157. 164.

78. —_, A suggested new "seismological" latitude, ibid. vol. 4 (1937) pp. 159-

79. H. Jeffreys, The ellipticity correction to the $P$ table, ibid. vol. 4 (1937) pp. 165184.

80. K. Uller, Entwicklung des Wellenbegriffes. I-IX, Gerlands Beiträge zur Geophysik vol. 18 (1927) pp. 398-414; vol. 24 (1929) pp. 309-334; vol. 26 (1930) pp. 199- 
238; vol. 27 (1930) pp. 71-101; vol. 29 (1931) pp. 252-266; vol. 31 (1931) pp. 40-82; vol. 41 (1934) pp. 225-249; vol. 43 (1934) pp. 289-295; vol. 47 (1936) pp. 299-320.

81. K. Sezawa, On the decay of waves in visco-elastic solid bodies, Bulletin of the Earthquake Research Institute, Tokyo Imperial University vol. 3 (1927) pp. 43-53.

82. - Notes on the waves in visco-elastic solid bodies, ibid. vol. 10 (1932) pp. 20-22.

83. K. Sezawa and K. Kanai, Damping of periodic visco-elastic waves with increasing focal distance. I, II, ibid. vol. 16 (1938) pp. 491-502; vol. 17 (1939) pp. 9-26.

84. H. Jeffreys, The viscosity of the earth, Monthly Notices of the Royal Astronomical Society of London, Geophysical Supplement vol. 1 (1926) pp. 412-424.

85. - Damping in bodily seismic waves, ibid. vol. 2 (1931) pp. 318-323.

86. B. Gutenberg and H. Schlechtweg, Viskosität und innere Reibung fester Körper, Physikalische Zeitschrift vol. 31 (1930) pp. 745-752.

87. F. D. Murnaghan, Finite deformations of an elastic solid, Amer. J. Math. vol. 59 (1937) pp. 235-260.

88. F. Birch, The effect of pressure upon the elastic parameters of isotropic elastic solids, according to Murnaghan's theory of finite strain, Journal of Applied Physics vol. 9 (1938) pp. 279-288.

89. - The variation of seismic velocities within a simplified earth model, in accordance with the theory of finite strain, Bulletin of the Seismological Society of America vol. 29 (1939) pp. 463-479.

90. M. A. Biot, Non-linear theory of elasticity and the linearized case for a body under initial stress, London, Edinburgh and Dublin Philosophical Magazine and Journal of Science (7) vol. 27 (1939) pp. 468-489.

91. - The influence of initial stress on elastic waves, Journal of Applied Physics vol. 11 (1940) pp. 522-530.

92. M. P. Rudzki, Parametrische Darstellung der elastischen Welle in anisotropen Medien, Bulletin de l'Académie des Sciences de Cracovie, 1911, pp. 503-506.

93. - Sur la propagation d'une onde élastique superficielle dans un milieu transversalement isotrope, ibid. 1912, pp. 47-58. (This and the preceding reference taken from a review by F. Pockels in Gerlands Beiträge zur Geophysik vol. 12 (1913) Kleine Mitteilungen pp. 75-79.)

Balch Graduate School of the Geological Sciences

California Institute of Technology

Contribution No. 340 